\title{
MODELING OF DOPPLER FREQUENCY SHIFT IN MULTIPATH RADIO CHANNELS
}

\author{
M.S. Penzin, N.V. Ilyin
}

Institute of Solar-Terrestrial Physics SB RAS, Irkutsk, Russia,penzin.maksim@gmail.com

We discuss the modeling of propagation of a quasi-monochromatic radio signal, represented by a coherent pulse sequence, in a non-stationary multipath radio channel. In such a channel, signal propagation results in the observed frequency shift for each ray (Doppler effect). The modeling is based on the assumption that during propagation of a single pulse a channel can be considered stationary. A phase variation in the channel transfer function is shown to cause the observed frequency shift in the received signal. Thus, instead of measuring the Doppler frequency shift, we can measure the rate of variation in the mean phase of one pulse relative to another. The modeling is carried out within the framework of the method of normal waves. The method enables us to model the dynamics of the electromagnetic field at a given point with the required accuracy. The modeling reveals that a local change in ionospheric conditions more severely affects the rays whose reflection region is in the area where the changes occur.

Keywords: Doppler effect, method of normal waves, modeling, non-stationary radio channel.

\section{INTRODUCTION}

At present, the most commonly adopted method of measuring the Doppler frequency shift is the spectral analysis [Baddeley et al., 2005; Bianchi, Altadill, 2005; Petrova et al., 2009]. With this approach for a quasi-monochromatic signal in the case of a stationary medium and constant velocity of a transmitter or receiver, we obtain a pronounced peak whose position is determined by a frequency shift (classical Doppler effect).

For a non-stationary medium and time-varying velocity of a receiver or transmitter, we get the socalled Doppler spectrum, thus losing some details in the temporal behavior of the frequency shift, hereinafter referred to as fine structure of the Doppler effect. Notice that the Doppler spectrum has different interpretations depending on conditions of a problem to solve.

An example of such a non-stationary medium is the ionosphere. When utilizing spectral methods to measure the Doppler frequency shift in a quasi-monochromatic signal that propagated through the non- 
stationary ionospheric radio channel, we are limited by two factors: dispersion precludes the formation of a too short signal, and nonstationarity hinders the analysis of too long signals. Typical values of Doppler (parametric) frequency shifts of the quasi-monochromatic radio signal in an HF band are hertzes or fractions of a hertz. Measuring similar values requires signals lasting up to tens of seconds, but radiochannel nonstationarity imposes constraints on the use of such signals.

Another approach allowing us to measure the fine structure of frequency shift is the analysis of a signal phase. For a single-path signal it is a fairly easy task. However, as a continuous signal propagates through a multipath radio channel, phase behavior becomes rather complicated, representing the behavior of a sum of ray phases and not of the phase of an individual ray. The problem of separating rays of such a signal is incorrect and generally unsolved.

Instead of the continuous signal, we can use a pulsed signal that can be split into separate rays at a receiving point with respect to delays. Radio-channel dispersion and nonstationarity slightly affect the pulses whose bandwidth is much narrower than coherence bandwidth of the channel (about hundreds of $\mathrm{kHz}$ [Ivanov et al., 2006]), and their length is comparable to propagation time and is much shorter than the characteristic time for changes in parameters of the radio channel. A radio channel can be considered stationary during propagation of one pulse of this type, but single pulses do not allow us to measure the rate of change in ionospheric parameters. In this case, we can register either a pulse-train spectrum [Batukhin et al., 2000] that is discrete or an amplitude and phase of each pulse with subsequent analysis of their temporal variations.

In the general case, temporal variations in parameters of the ionospheric radio channel cause changes in characteristics of separate pulses. It is, however, easy to establish that during spectral measurements of a coherent pulse sequence, only phase variations in the channel transfer function (that determines the mean phase of an amplitude-modulated pulse) change the position of the central spectral line in the discrete spectrum. In this case, amplitude variations or delays do not lead to shifts of the central line. Amplitude variations cause deformation of spectral lines; and delay variations produce a small change in distance between spectral lines, leaving the position and shape of the central line unchanged.

Thus, in the case of small variations in ionospheric parameters, signal phase and amplitude vary continuously from pulse to pulse; amplitude variations may be ignored. The phase variation rate can be interpreted as the Doppler frequency shift [Barnes, 1992; Cohen, 1995].

Accordingly, employing a model of transfer function of the multipath radio channel whose parameters vary slowly and continuously with time, we can register phases of certain pulses corresponding to individual rays. Phase variations in a received signal allow us to trace the Doppler frequency shift for each ray, using the coherent pulse sequence. 


\section{PROPAGATION OF THE COHERENT PULSE SEQUENCE THROUGH THE RADIO CHANNEL}

For simplicity, we consider narrowband amplitude-modulated pulses with a carrier frequency $\omega_{0}$ and amplitude $a(t)$. The pulses repeat themselves at a time interval $T$.

A particular pulse can be represented as

$u(t)=a(t) \cos \left(\omega_{0} t\right)$.

The coherent sequence of generated pulses $(0)$

$$
F(\omega)=U(\omega) \sum_{k=0}^{K-1} \exp (i \omega k T),
$$

where $K$ is the number of pulses.

For simplicity, we assume that $\omega_{0} T=2 \pi n$ with $n$ being an integer. Then pulse-train spectrum (0) can be written as

$$
F(\omega)=U(\omega) \sum_{k=0}^{K-1} \exp (i \omega k T)
$$

where $U(\omega)=\left(A\left(\omega-\omega_{0}\right)+A\left(\omega+\omega_{0}\right)\right) / 2, A(\omega)$ is the $a(t)$ spectrum. In this case,

$$
\left|\sum_{k=0}^{K-1} \exp (i \omega k T)\right|^{2}=\left(\frac{\sin \frac{\omega T K}{2}}{\sin \frac{\omega T}{2}}\right)^{2} .
$$

Equation (4) shows that as the number of terms increases the sum of exponents tends to the sum of $\delta$-functions. The coherent signal sequence is needed in order that exponents in the sum have no phase shifts. In fact, the finite sum differs from the $\delta$-function and takes the form of narrow peaks repeating themselves with a period of $2 \pi / T$ with lower peaks grouped together in the near vicinity. Thus, the spectrum of coherent pulse sequence becomes discrete. Yet the spectrum envelope in the vicinity of $\omega= \pm \omega_{0}$ is equal to the spectrum of a single pulse $A(\omega)$.

As the pulse-repetition period decreases, spectral lines move apart; and as it increases, the lines become closer to each other.

When the pulses begin to merge, i.e. their repetition period becomes shorter or equal to the duration of a certain pulse, there remains only one line. If the envelope of a continuous signal varies slightly, the line turns into a very sharp peak corresponding to a monochromatic signal. Thus, the continuous monochromatic signal can be considered as a coherent sequence of pulses with a repetition period being shorter or equal to their duration. 
Let us describe propagation of the pulse sequence through a single-path quasi-stationary channel whose parameters slowly vary with time. By the term quasi-stationary we mean that such a channel in a signal band for the propagation time of $k$ th pulse can be considered stationary and can be characterized by a transfer function.

$$
\begin{aligned}
& H_{k}(\omega)=\left|H_{k}(\omega)\right| e^{i \Phi_{k}(\omega)} \approx \\
& \approx H_{0 k}\left(1+\gamma_{0 k}\left(\omega-\omega_{0}\right)\right) e^{i \Phi_{0 k}+i \tau_{0 k}\left(\omega-\omega_{0}\right)},
\end{aligned}
$$

where $H_{0 k}=\left|H_{k}\left(\omega_{0}\right)\right|$ is the modulus of transfer function at the carrier frequency; $\tau_{0 k}=\Phi_{k}^{\prime}\left(\omega_{0}\right)$ is the group delay of the pulsed signal equal to the frequency derivative of transfer function phase; $\gamma_{0 k}=\left.\left(\ln \left|H_{k}(\omega)\right|\right)^{\prime}\right|_{\omega=\omega_{0}}$ is the logarithmic frequency derivative of transfer function modulus characterizing pulse distortions in the first-order bandwidth; $\Phi_{0 k}=\Phi_{k}\left(\omega_{0}\right)$ is the transfer function phase at the carrier frequency, subsequently referred to as phase.

The spectrum of coherent pulse sequence ( 0 ) having passed through the radio channel takes the form

$$
F(\omega)=U(\omega) \sum_{k} H_{k}(\omega) \exp (i \omega k T)
$$

For the stationary radio channel, $H_{0 k}=H_{0}, \tau_{0 k}=\tau_{0}, \gamma_{0 k}=\gamma_{0}, \Phi_{0 k}=\Phi_{0}$. Then

$$
F(\omega)=U_{0}(\omega) \sum_{k} \exp (i \omega k T)
$$

Here we introduce a designation

$$
U_{0}(\omega)=U\left(\omega_{0}\right) H_{0}\left(1+\gamma_{0}\left(\omega-\omega_{0}\right)\right) e^{i \Phi_{0}+i \tau_{0}\left(\omega-\omega_{0}\right)} .
$$

Let us consider the effects of variations in each transfer function parameter on a received signal.

First, we analyze phase variations. Let the phase vary linearly from pulse to pulse, i.e. $\Phi_{k}=\Phi_{0}+k \Delta \Phi$. Then the spectrum of the adopted sequence can be written as

$$
F(\omega)=U_{0}(\omega) \sum_{k} \exp \left[i\left(\omega+\frac{\Delta \Phi}{T}\right) k T\right] .
$$

Figure 1 illustrates spectrum (8). From now on, spectra are presented without considering the envelope $U_{0}(\omega)$. Along the x-axis is the deviation from the carrier frequency $\omega_{0}$. The dashed line indicates the spectrum of pulse sequence in the stationary case; the solid line, in the non-stationary one.

Thus, the apparent frequency shift in the adopted sequence $\Delta \omega=\Delta \Phi / T$. This shift can be seen in Figure 1.

Let us now examine the influence of a group delay shift. Let the group delay also vary linearly from pulse to pulse $\tau_{k}=\tau_{0}+k \Delta \tau$. The spectrum can then be written as:

$$
F(\omega)=U_{0}(\omega) \sum_{k} \exp \left[i(T+\Delta \tau)\left(\omega-\omega_{0}\right) k\right] .
$$


From (4) and (9) it follows that only the distance between spectral lines varies, whereas shape and position of the spectral line at $\omega_{0}$ remain unchanged. This can be seen in Figure 2 that depicts spectrum (9).

Now let the amplitude vary linearly, $H_{0 \mathrm{k}}=H_{0}+\Delta H k$, then

$$
F(\omega)=U_{0}(\omega) \sum_{k}\left(1+\frac{\Delta H k}{H_{0}}\right) \exp (i \omega k T) .
$$

Figure 3 shows that the small linear amplitude variation leads only to an insignificant broadening of spectral line without causing spectral line shifts.

Lastly, we consider the effect of $\gamma_{0 k}=\gamma_{0}+\Delta \gamma k$ :

$$
F(\omega)=U_{0}(\omega) \sum_{k}\left(1+\frac{\Delta \gamma\left(\omega-\omega_{0}\right) k}{1+\gamma_{0}\left(\omega-\omega_{0}\right)}\right) \times \exp (i \omega k T)
$$

Figure 4 indicates that the linear variation in $\gamma_{0}$ does not lead to shifts in spectral peaks either, but changes their shapes.

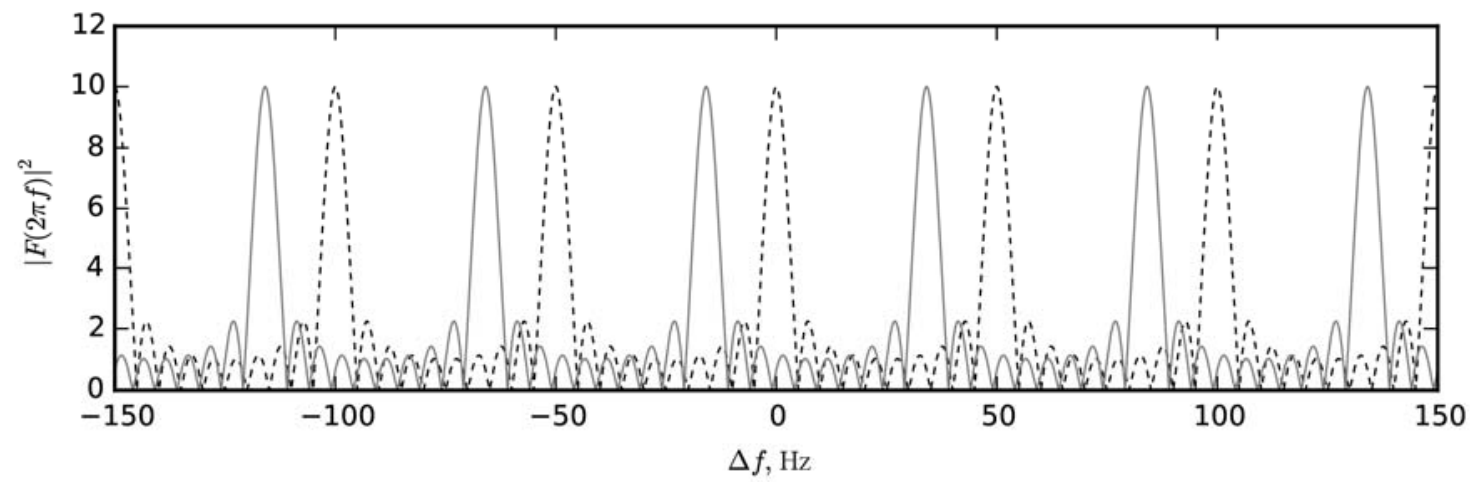

Figure 1. Spectrum of coherent pulse sequence with linearly varying $\Phi_{0}$

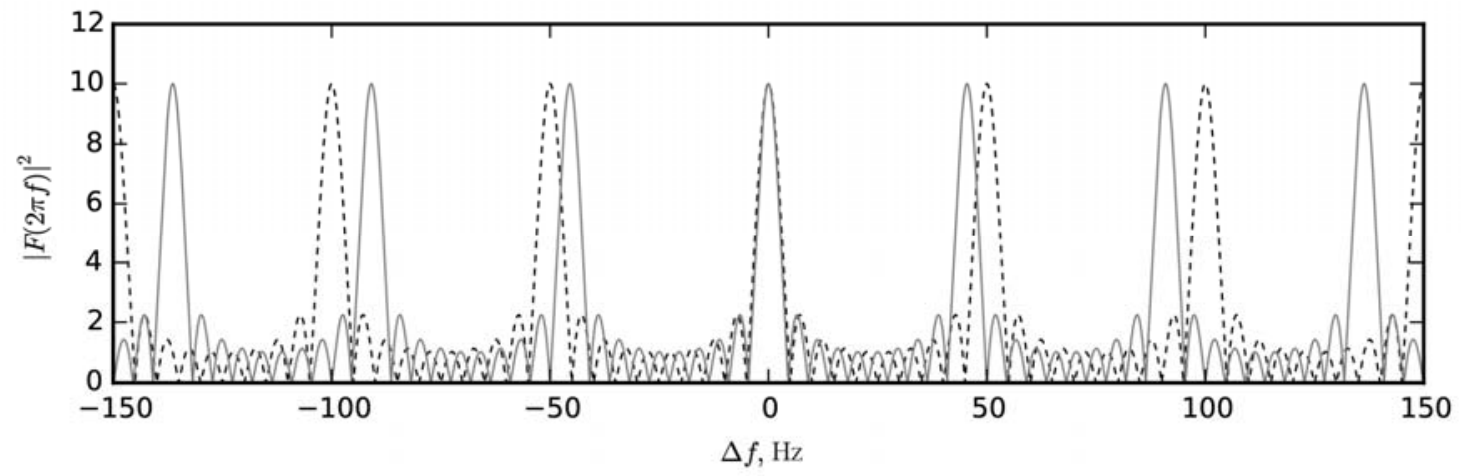

Figure 2. Spectrum of coherent pulse sequence with linearly varying $\tau_{0}$ 


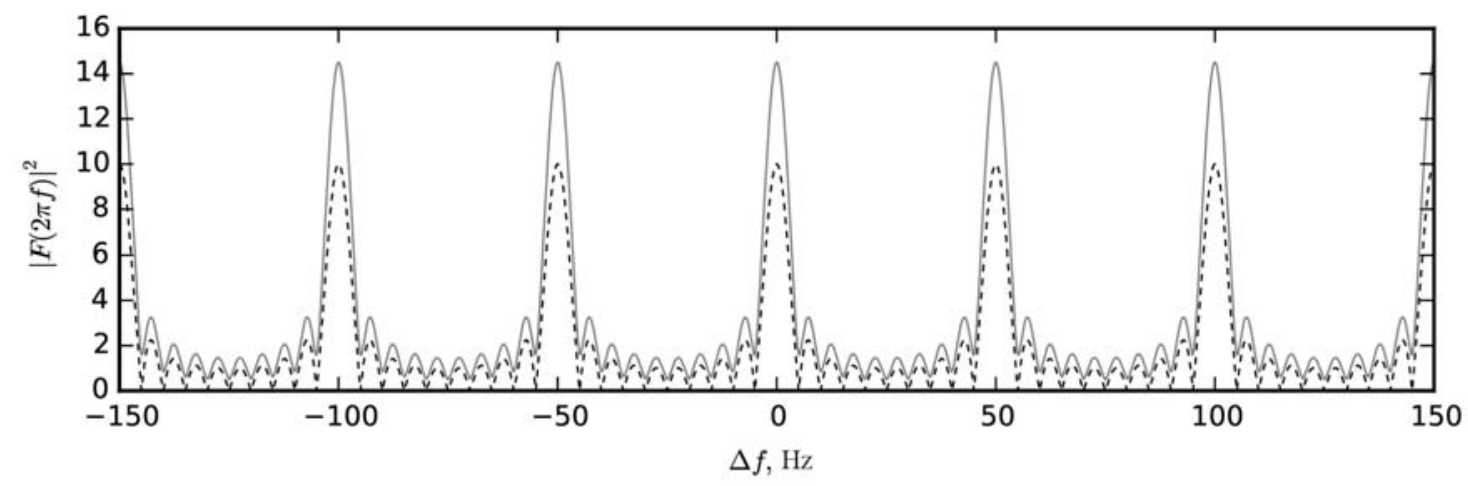

Figure 3. Spectrum of coherent pulse sequence with linearly varying $H_{0}$

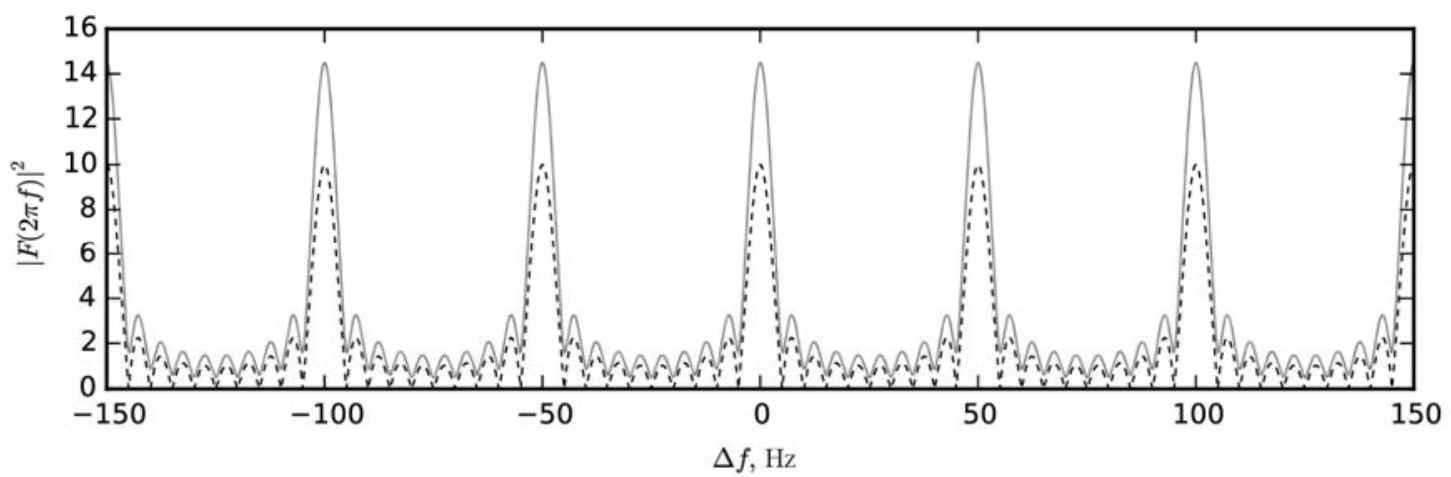

Figure 4. Spectrum of coherent pulse sequence with linearly varying $\gamma_{0}$

Thus, the observed shift in the spectrum of the quasi-monochromatic signal is induced only by the temporal variation in the transfer function phase. Yet the phase is also the most rapidly changing quantity.

A multipath radio channel is a sum of single-path channels; therefore a transfer function is also a sum of transfer functions of separate rays.

Let there be several rays, each represented at a receiving point as

$u_{i}(t)=a_{i} \cos \left(\omega_{0} t+\phi_{i}\right)+b_{i} \sin \left(\omega_{0} t+\phi_{i}\right)$.

Then their sum takes the form

$$
u(t)=\cos \left(\omega_{0} t\right)\left[\sum_{i}\left(a_{i} \cos \phi_{i}+b_{i} \sin \phi_{i}\right)\right]+\sin \left(\omega_{0} t\right)\left[\sum_{i}\left(-a_{i} \sin \phi_{i}+b_{i} \cos \phi_{i}\right)\right] .
$$

Hence, a signal at a receiving point is a vector sum of amplitudes of separate rays. In the stationary case, we have a purely harmonic oscillation with an amplitude and phase. In the non-stationary case, parameters of each of the rays continuously vary with time. This results in a continuous variation in their sum. Yet parameters of each ray vary with variations in parameters of a medium. Each of the rays is a quasi-monochromatic signal, but a signal composed of the sum of these rays at a receiving point is noise-like due to interference of these rays. 
When modeling the Doppler frequency shift, we account only for the phase variation in the transfer function of the channel, ignoring other parameters. Thus, the simplest algorithm for measuring the Doppler frequency shift can be represented as follows:

1. A coherent pulse sequence is generated.

2. At a receiving point, we separate pulses according to delays and correlate them with particular channels.

3. For each pulse, we determine its mean phase, thus obtaining a phase-time diagram for each channel.

4. We transform the phase-time diagram to a frequency-time one, operating on the premise that the frequency is a time derivative of phase.

Notice that the pulse phase can be determined only with accuracy to $2 \pi n$ with $n$ being an integer. In order to maintain continuity and unambiguity in the determination of phase, we should impose a condition such that the phase difference between pulses should not exceed $\pi$ in modulus. In the HF ionospheric radio channel, the Doppler frequency shift does not normally exceed $10 \mathrm{~Hz}$. Given the maximum phase difference between pulses and the maximum rate of frequency change, we can obtain a restriction on the pulse-repetition period $T$ :

$$
T \leq \frac{1}{2 f_{\max }}
$$

where $f_{\max }$ is an expected maximum frequency shift. For $f_{\max }$ of about $10 \mathrm{~Hz}, T$ should not exceed $0.05 \mathrm{~s}$.

\section{MODELLING}

We model the Doppler shift, using the method of normal waves. This approach (unlike the geometrical one) allows us to model field variations at a certain point of space, thus making it possible to trace smooth variations in pulse phases.

In the method of normal waves, the channel transfer function [Kurkin et al., 1981] can be written as

$$
H(\omega)=\sum_{n} \frac{A_{n}}{r r_{0}^{2} \sqrt{\sin \theta}} I_{n}\left(r_{0}, 0\right) I_{n}(r, \theta) \exp \left[i k a \int_{0}^{\theta} \gamma_{n}\left(\theta^{\prime}\right) d \theta^{\prime}\right],
$$

where $A_{n}$ is a system coefficient, $r_{0}$ is a height of a transmitter, $r$ and $\theta$ are coordinates of a receiver, $I_{n}$ are excitation coefficients, $a$ is the Earth radius, $k$ is a wave number, $\gamma_{n}$ is an eigen value of radial operator.

For fixed transmitting and receiving points in the case of a quasi-monochromatic signal at the carrier frequency $\omega_{0}$, the sum are split into several sums corresponding to rays in geometrical optics [Potekhin, Orlov, 1981]. This allows us to utilize terminology of geometrical optics.

As an example, we take the ionosphere to be the mutipath radio channel, presenting its normalized electron-density profile in the form of two quasi-parabolas [Kurkin et al., 1981]: 


$$
q(h)=\left\{\begin{array}{cl}
0, & h<h_{\mathrm{b}}, \\
d_{1}\left(1-\frac{h_{\mathrm{b}}+a}{h+a}\right)^{2}, & h_{\mathrm{b}} \leq h \leq h_{0}, \\
1-d_{2}\left(\frac{h_{\mathrm{m}}+a}{h+a}-1\right)^{2}, & h_{0} \leq h \leq h_{\mathrm{m}},
\end{array}\right.
$$

where $a$ is the Earth radius, $h$ is a height, $h_{\mathrm{b}}$ is the height of the beginning of the ionosphere, $h_{\mathrm{m}}$ is the maximum ionization height, $h_{0}$ is a matching point, and $d_{1}$ and $d_{2}$ are defined by

$$
d_{1}=\left[\left(1-\frac{h_{\mathrm{b}}+a}{h_{0}+a}\right)\left(1-\frac{h_{\mathrm{b}}+a}{h_{\mathrm{m}}+a}\right)\right]^{-1}, d_{2}=\left[\left(\frac{h_{\mathrm{m}}+a}{h_{\mathrm{b}}+a}-1\right)\left(\frac{h_{\mathrm{m}}+a}{h_{0}+a}-1\right)\right]^{-1} .
$$

The critical frequency varies with time according to the law

$$
f_{\text {cr }}=f_{\text {ocr }}\left[1+a_{f} \cos \left(\frac{2 \pi t}{T}\right) \sin \left(\frac{10 \pi t}{T}\right)\right]
$$

where $a_{f}=0.01$ and $T=7200 \mathrm{~s}$.

The position of the point at the beginning of the ionosphere also changes:

$$
h_{\mathrm{b}}=h_{0 \mathrm{~b}}\left[1+0.5 a_{h}-0.5 a_{h} \cos \left(\frac{2 \pi t}{T}\right)\right] \text {, }
$$

where $a_{h}=0.000785$.

We have selected the following initial values of ionospheric parameters: the height of the lower boundary of the ionosphere is $90 \mathrm{~km}$, the matching point is $195 \mathrm{~km}$, the maximum ionization height is $300 \mathrm{~km}$, and the critical frequency is $6 \mathrm{MHz}$. Through this radio channel, we run a sequence of coherent pulses with a $10 \mathrm{MHz}$ carrier signal frequency and $120 \mu$ s pulse duration. The distance between transmitting and receiving points is $3500 \mathrm{~km}$.

We modeled propagation of one pulse through the ionospheric radio channel for each moment of time. At the receiving point, after demodulation, we separated the pulses with respect to delays and for each pulse we found mean amplitude and phase. The pulse-repetition period was taken to be $0.05 \mathrm{~s}$.

Phases of particular rays obtained from the modeling of propagation of the coherent pulse sequence through the ionospheric radio channel are shown in Figure 5. Four rays arrive at the receiving point: two double-hop and two triple-hope ones. It is apparent that lower rays are more sensitive to variations in parameters of the lower ionosphere where regions of their reflection are located. At the same time, upper rays are more sensitive to variations in characteristics of the upper ionosphere (in this case, to critical frequency change). Yet in both the cases, double-hop rays appear to be most sensitive. This behavior agrees well with the physical notion of the process. 
The Doppler frequency shift is defined as a phase variation rate (Figure 6). Thus, we can observe the fine structure of the Doppler frequency shift depending on variations in parameters of a medium.

Figure 7 presents the results of the modeling of propagation of a $10 \mathrm{MHz}$ continuous quasimonochromatic signal. Owing to strong interference and infinitesimality of frequency shifts, as indicated above, it is not always possible to determine the Doppler shift of a separate ray in the general case.

\section{CONCLUSION}

We have analyzed a method of measuring the Doppler frequency shift in multipath radio channels for each ray represented by a coherent pulse sequence. The method allows us to thoroughly monitor variations in radio channel parameters (fine structure).

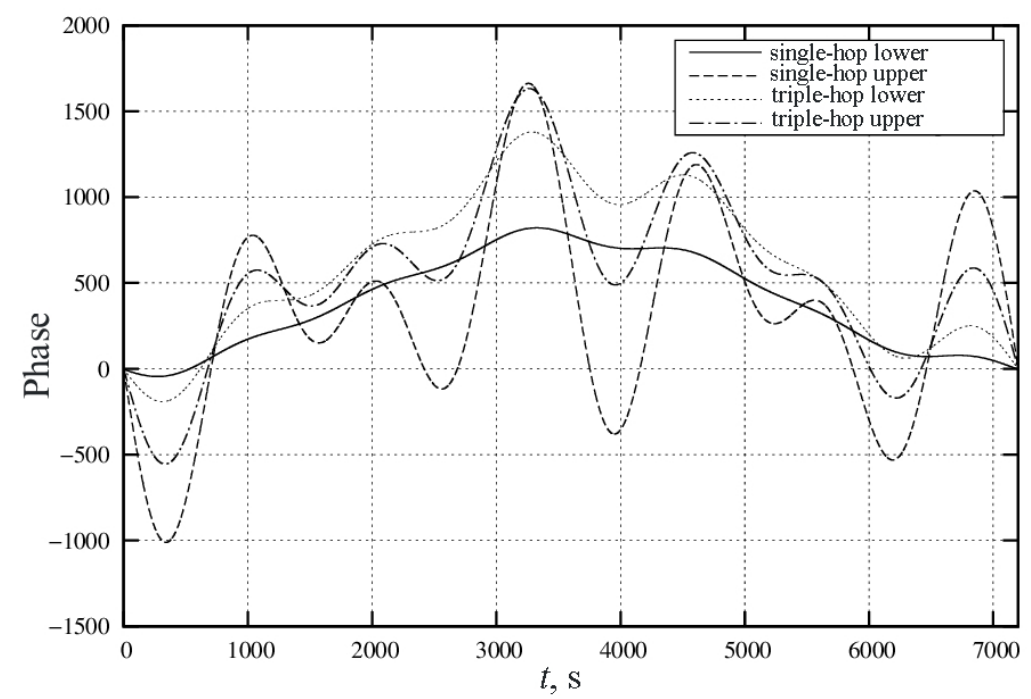

Figure 5. Phases of separate pulses

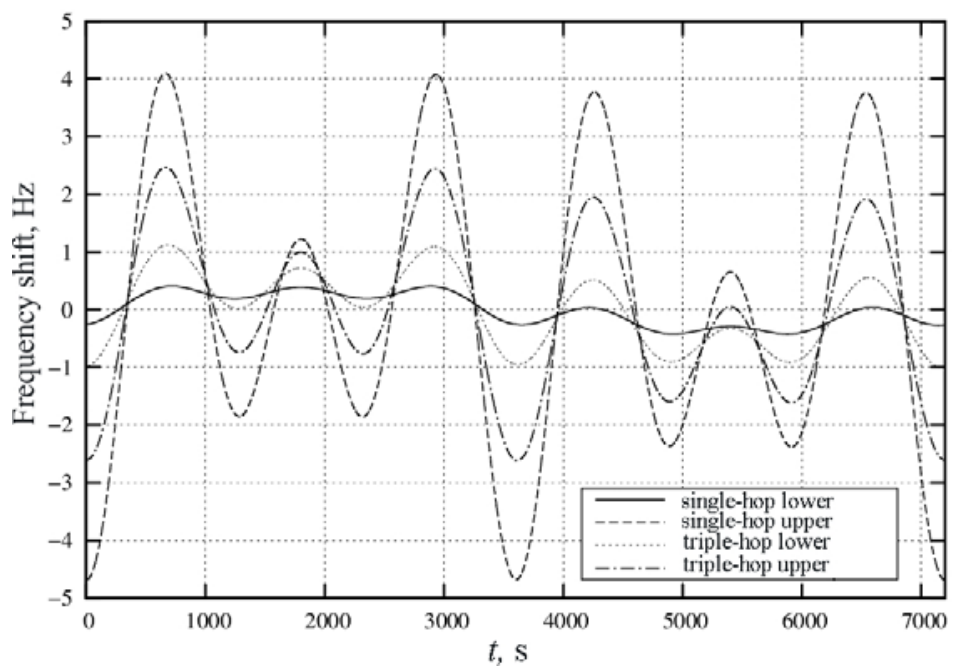

Figure 6. Doppler frequency shift in separate pulses 

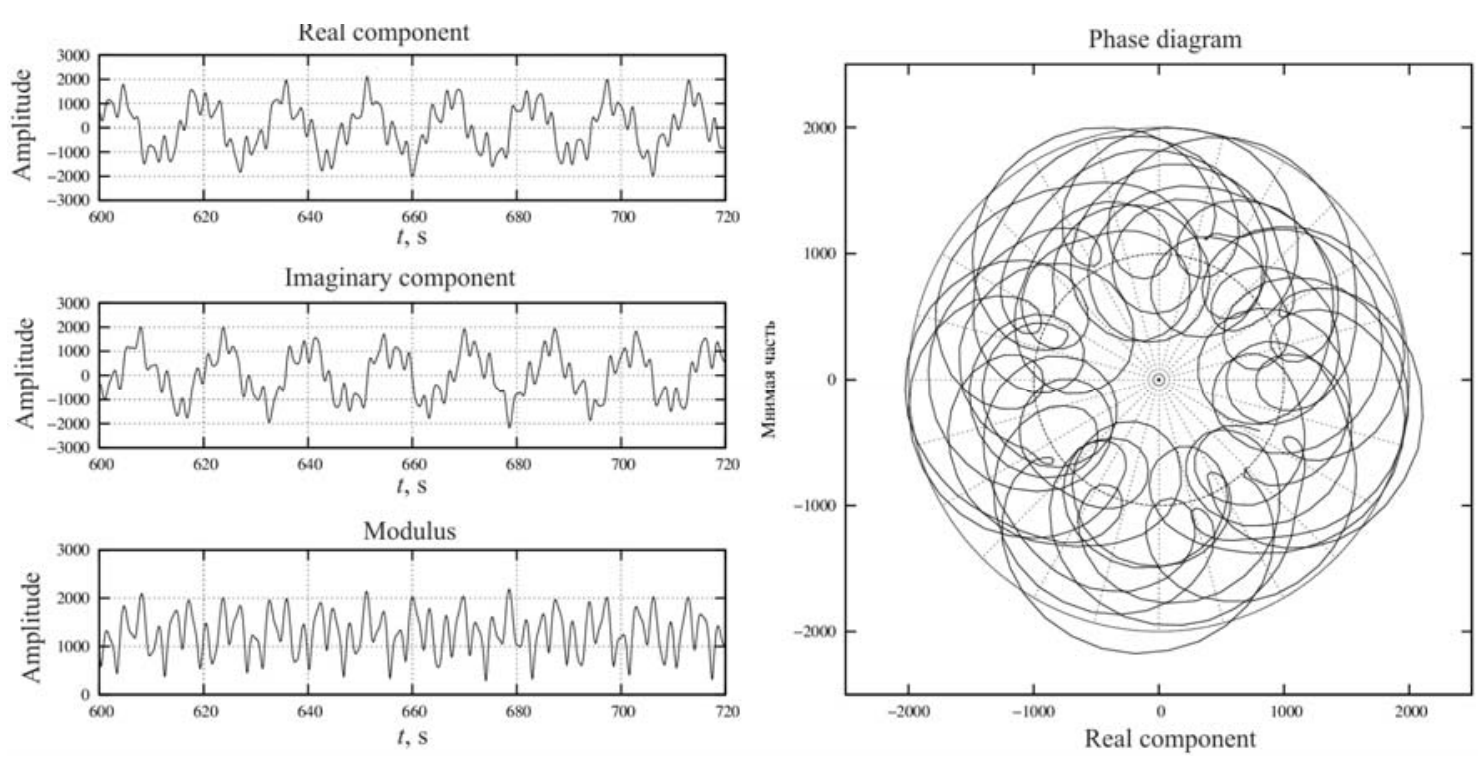

Figure 7. Continuous signal

We have shown that the greatest contribution to the Doppler frequency shift is made by phase variations in a channel transfer function.

We utilized this method to model propagation of the coherent pulse sequence in the simplest case of the plane-stratified ionosphere having parameters slowly varying with time. The modeling results indicate that variations in characteristics of a separate ray are caused by variations in ionospheric parameters in reflection regions. This qualitatively agrees with experimental data.

Thus, using a model of ionospheric dynamics, we can analyze phase and amplitude variations in separate rays. Yet variations in ray parameters are actually caused only by variations in ionospheric parameters in reflection regions. This can allow us to determine the nature of the Doppler frequency shift and to find out if there are any peculiarities in the manifestation of the Doppler effect typical for this model of ionospheric dynamics.

The work was funded by RFBR (No.13-05-00979-a).

\section{REFERENCES}

Baddeley L.J., Yeoman T.K., Wright D.M. HF Doppler sounder measurements of the ionospheric signatures of small scale ULF waves. Ann. Geophys. 2005, vol. 23, pp. 1807-1820.

Barnes A.E. The calculation of instantaneous frequency and instantaneous bandwidth (Short Note). Geophysics. 1992, vol. 57, no. 11, pp. 1520-1524.

Batukhin V.I., Ivanov V.A., Kolchev A.A., Rozanov S.Z. Measuring the Doppler frequency shift of particular rays using LFM ionosonde. Izvestiya vuzov. Radiofizika [Radiophysics and Quantum Electronics]. 2000, vol. 43, no. 12, pp. 1044-1054. (In Russian).

Bianchi C., Altadill D. Ionospheric Doppler measurements by means of HF-radar techniques. Ann. Geophys. 2005 , vol. 48 , no. 6 , pp. 989-993. 
Cohen L. Time-Frequency Analysis. New Jersey, Prentice Hall Ptr, 1995, 206 p.

Ivanov D.V., Yegoshin A.B., Ivanov V.A., Ryabova N.V. Informatsionno-Analiticheskaya Sistema dlya Issledovaniya Ionosfery i Kanalov Dekametrovoi Radiosvyazi [Information-Analytical System for Studying the Ionosphere and Channels of Decameter Radio Communication]. Yoshkar-Ola, Volga State University of Technology Publ., 2006, 256 p. (In Russian).

Kurkin V.I., Orlov I.I., Popov V.N. Method Normal'nykh Voln v Probleme Korotkovolnovoi Radiosvyazi [Method of Normal Waves in the Problem of Short-Wave Radio Communication]. Moscow, Nauka Publ., 1981, 124 p. (In Russian).

Petrova I.R., Bochkarev V.V., Latipov R.R. Application of HF Doppler measurements for the investigation of internal atmospheric waves in the ionosphere. Adv. Space Res. 2009, vol. 44, no. 6, pp. 685-692.

Potekhin A.P., Orlov I.I. Approximate formula of summation of a sequence of normal waves. Issledovaniya po geomagetizmu, aeronomii i fizike Solntsa [Research on geomagnetism, Aeronomy and Solar Physics]. Moscow, Nauka Publ., 1981, iss. 57, pp. 135-137. (In Russian). 